\title{
Parton Distribution Functions and One-Loop Matching
}

\author{
Xiangdong Ji \\ INPAC, Department of Physics and Astronomy, \\ Shanghai Jiao Tong University, Shanghai, 200240, P. R. China \\ Center for High-Energy Physics, Peking University, \\ Beijing, 100080, P. R. China \\ Maryland Center for Fundamental Physics, Department of Physics, \\ University of Maryland, College Park, Maryland 20742, USA \\ Jian-Hui Zhang* \\ INPAC, Department of Physics and Astronomy, \\ Shanghai Jiao Tong University, Shanghai, 200240, P. R. China \\ Institut für Theoretische Physik, Universität Regensburg, \\ D-93040 Regensburg, Germany \\ Yong Zhao \\ Maryland Center for Fundamental Physics, Department of Physics, \\ University of Maryland, College Park, Maryland 20742, USA \\ Published 29 February 2016
}

\begin{abstract}
We discuss recent developments on computing a light-cone parton distribution from the large momentum limit of the hadronic matrix element of a space-like correlator, and present the one-loop matching condition connecting these two quantities. The matching condition is useful for extracting the former quantity from the latter, which is calculable on the lattice.
\end{abstract}

Keywords: Light-cone parton distribution; quasi parton distribution; matching.

PACS numbers: 12.38.Bx, 12.38.Gc, 14.20.Dh

\section{Introduction}

Parton distribution functions are crucial quantities characterizing the internal structure of hadrons, and play an important role in describing high energy scattering

*Speaker.

This is an Open Access article published by World Scientific Publishing Company. It is distributed under the terms of the Creative Commons Attribution 3.0 (CC-BY) License. Further distribution of this work is permitted, provided the original work is properly cited. 
experiments involving hadrons. Although much effort has been devoted to extracting parton distributions from various experimental data, ${ }^{1-6}$ the computation of parton distributions from the underlying theory of strong interactions, quantum chromodynamics (QCD), has been a difficult task, due to their non-perturbative nature. Given the success of lattice QCD on studying non-perturbative phenomena in $\mathrm{QCD}$, one may wonder whether it can be used to compute the parton distributions. However, the parton distributions are defined in terms of non-local light-cone correlators, which are time-dependent and intrinsically Minkowskian, and therefore cannot be readily computed on the lattice.

Recently, it was shown that the light-cone gluon helicity contribution to the proton spin can be obtained as the large momentum limit of the proton matrix element of a time-independent correlator. ${ }^{7}$ As the matrix element of the time-independent correlator is calculable on the lattice, this opens up a new possibility of computing the light-cone gluon helicity contribution to proton spin using lattice QCD. A similar strategy also applies to parton distributions and other quantities defined on the light-cone,$^{8-15}$ where they can be obtained as the large momentum limit of the hadronic matrix element of suitable space-like correlators. Let us take the unpolarized quark distribution as an example. The light-cone unpolarized quark distribution can be obtained from the large momentum limit of the following matrix element $^{8}$

$\tilde{q}\left(x, \Lambda, P^{z}\right)=\int_{-\infty}^{\infty} \frac{d z}{4 \pi} e^{i z k^{z}}\left\langle P\left|\bar{\psi}\left(0,0_{\perp}, z\right) \gamma^{z} \exp \left(-i g \int_{0}^{z} d z^{\prime} A^{z}\left(0,0_{\perp}, z^{\prime}\right)\right) \psi(0)\right| P\right\rangle$,

which may be called a quasi quark distribution. In the quasi quark distribution, the quark fields are separated along the spatial $z$-axis instead of being separated along the light-cone, $x=k^{z} / P^{z}$ is the longitudinal momentum fraction, $|P\rangle$ is the nucleon state with four-momentum $P^{\mu}=\left(P^{0}, 0,0, P^{z}\right)$, and $\Lambda$ is the momentum cut-off to regulate potential UV divergences. Using the standard operator product expansion, the above quasi quark distribution can be shown to approach the light-cone one up to power suppressed corrections in the large nucleon momentum limit. ${ }^{8}$ The operator appearing in Eq. (1) is time-independent, its matrix element can therefore be simulated on a lattice for any $P^{z} \ll 1 / a \sim \Lambda$, where $a$ is the lattice spacing. The result is not the light-cone distribution extracted from the experimental data, $q(x, \mu)$ (scheme-dependent, usually in the $\overline{\mathrm{MS}}$ scheme and $\mu$ indicates the renormalization scale), but one can recover the latter from the former by a matching condition of the following type 8,10

$$
\tilde{q}\left(x, \Lambda, P^{z}\right)=\int \frac{d y}{|y|} Z\left(\frac{x}{y}, \frac{\Lambda}{P^{z}}, \frac{\mu}{P^{z}}\right) q(y, \mu)+\mathcal{O}\left(M^{2} /\left(P^{z}\right)^{2}\right)
$$

for a large $P^{z}$, where we have limited ourselves to the so-called non-singlet quantities such as up minus down flavors, so that the gluon contribution can be ignored. The correction terms are in power of $M / P^{z}$, where $M$ is a QCD scale such as the hadron mass. The difference between $\tilde{q}$ and $q$ is that the former is for finite but large 
momentum while the latter is for infinite momentum, thus the IR behavior of the distribution should not change when moving from one frame to the other, and the matching factor $Z$ captures only the UV behavior and is thus entirely perturbative. One can also view the above equation as a factorization of the quasi distribution into the light-cone distribution and a hard coefficient.

In the following, we show that a matching condition of the type in Eq. (2) can indeed be constructed, and present the one-loop matching factors connecting the quasi and the light-cone unpolarized quark distribution in the non-singlet case.

\section{One-Loop Correction and Factorization for Unpolarized Quark Distribution}

The one-loop computation for the non-singlet quark distribution is similar to QED since the non-Abelian property does not enter in the non-singlet case. At tree-level, the quasi distribution yields the same result as the light-cone one

$$
\tilde{q}^{(0)}(x)=q^{(0)}(x)=\delta(1-x) .
$$

The one-loop calculation can in principle be carried out in any gauge since the result is gauge invariant. A convenient choice is the axial gauge $A^{z}=0$, since in this gauge the gauge link in Eq. (1) becomes unity, and the relevant Feynman diagrams also become very simple, as shown in Fig. 1. The non-local operator is depicted as a dashed line.

The one-loop diagrams generate the following result

$$
\tilde{q}\left(x, \Lambda, P^{z}\right)=\left(1+\tilde{Z}_{F}^{(1)}\left(\Lambda, P^{z}\right)\right) \delta(x-1)+\tilde{q}^{(1)}\left(x, \Lambda, P^{z}\right)+\cdots,
$$

where we have included the tree-level result, $\tilde{q}^{(1)}\left(x, \Lambda, P^{z}\right)$ comes from the gluonexchange diagram, and $\tilde{Z}_{F}^{(1)}\left(\Lambda, P^{z}\right)$ comes from the self energy diagram.

In the finite momentum frame, the one-loop diagrams yield the following result for the quasi-distribution at a fixed $x$

$$
\begin{aligned}
& \tilde{q}^{(1)}\left(x, \Lambda, P^{z}\right) \\
&=\frac{\alpha_{S} C_{F}}{2 \pi} \begin{cases}\frac{1+x^{2}}{1-x} \ln \frac{x}{x-1}+1+\frac{\Lambda}{(1-x)^{2} P^{z}}, & x>1, \\
\frac{1+x^{2}}{1-x} \ln \frac{\left(P^{z}\right)^{2}}{m^{2}}+\frac{1+x^{2}}{1-x} \ln \frac{4 x}{1-x}-\frac{4 x}{1-x}+1+\frac{\Lambda}{(1-x)^{2} P^{z}}, & 0<x<1, \\
\frac{1+x^{2}}{1-x} \ln \frac{x-1}{x}-1+\frac{\Lambda}{(1-x)^{2} P^{z}}, & x<0,\end{cases}
\end{aligned}
$$
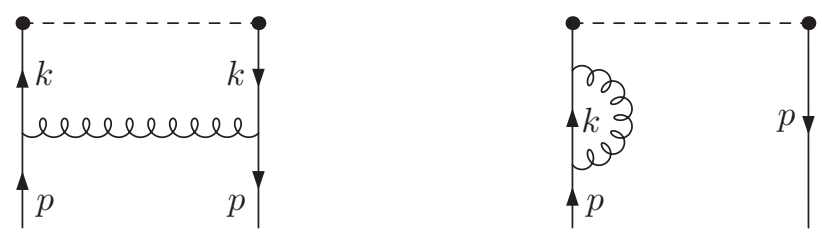

Fig. 1. One-loop corrections to quasi quark distribution. 
and

$$
\begin{aligned}
& \tilde{Z}_{F}^{(1)}\left(\Lambda, P^{z}\right)=\frac{\alpha_{S} C_{F}}{2 \pi} \\
& \quad \times \int d y \begin{cases}-\frac{1+y^{2}}{1-y} \ln \frac{y}{y-1}-1-\frac{\Lambda}{(1-y)^{2} P^{z}}, & y>1, \\
-\frac{1+y^{2}}{1-y} \ln \frac{\left(P^{z}\right)^{2}}{m^{2}}-\frac{1+y^{2}}{1-y} \ln \frac{4 y}{1-y}+\frac{4 y^{2}}{1-y}+1-\frac{\Lambda}{(1-y)^{2} P^{z}}, & 0<y<1, \\
-\frac{1+y^{2}}{1-y} \ln \frac{y-1}{y}+1-\frac{\Lambda}{(1-y)^{2} P^{z}}, & y<0,\end{cases}
\end{aligned}
$$

where we have introduced a quark mass $m$ to regularize the collinear divergences. One can easily check vector current conservation $\int_{-\infty}^{\infty} d x \tilde{q}\left(x, \Lambda, P^{z}\right)=1$ from the above results. It is interesting to note that the results do not vanish in the regions $x>1$ and $x<0$. The physics behind this is transparent: when the parent particle has a finite momentum $P^{z}$, the constituent parton can have momentum larger than $P^{z}$, and even negative. This is very different from the infinite momentum frame result, where the momentum fraction is restricted to $-1<x<1$ (At one-loop level, one has just $0<x<1$ in a quark target, but there are contributions for $-1<x<0$ at two-loop level). Moreover, there is no logarithmic UV divergence in $\tilde{q}^{(1)}$. Instead there is a logarithmic dependence on $P^{z}$ in the region $0<x<1$. This logarithmic dependence can be transformed into the renormalization scale dependence of the light-cone quark distribution by the matching condition that will be presented below. Also note that the soft divergences in the above results cancel, but the collinear divergences remain.

With the same regularization, one can calculate the light-cone quark distribution by taking the limit $P^{z} \rightarrow \infty$ before UV regularization. This leads to the following result

$$
q(x, \Lambda)=\left(1+Z_{F}^{(1)}(\Lambda)+\ldots\right) \delta(x-1)+q^{(1)}(x, \Lambda)+\cdots
$$

with

$$
q^{(1)}(x, \Lambda)=\frac{\alpha_{S} C_{F}}{2 \pi} \begin{cases}0, & x>1 \text { or } x<0, \\ \frac{1+x^{2}}{1-x} \ln \frac{\Lambda^{2}}{m^{2}}-\frac{1+x^{2}}{1-x} \ln (1-x)^{2}-\frac{2 x}{1-x}, & 0<x<1,\end{cases}
$$

and

$$
Z_{F}^{(1)}(\Lambda)=\frac{\alpha_{S} C_{F}}{2 \pi} \int d y \begin{cases}0, & y>1 \text { or } y<0 \\ -\frac{1+y^{2}}{1-y} \ln \frac{\Lambda^{2}}{m^{2}}+\frac{1+y^{2}}{1-y} \ln (1-y)^{2}+\frac{2 y}{1-y}, & 0<y<1\end{cases}
$$

where the integrand of $Z_{F}^{(1)}(\Lambda)$ is exactly opposite to that of $q^{(1)}(x, \Lambda)$, indicating the quark number conservation at one-loop. If dimensional regularization is used for the UV divergence, the results $q^{(1)}(x, \mu)$ and $Z_{F}^{(1)}(\mu)$ (with $\mu$ the renormalization scale) are slightly different, and can be obtained from the above ones by making the replacement $\ln \Lambda^{2} \rightarrow 1 / \epsilon_{U V}-\gamma_{E}+\ln 4 \pi \mu^{2}$. This result agrees with that derived 
from the light-cone definition of quark distribution. Note that the collinear or mass singularity is the same as in the quasi quark distribution. This shows that at oneloop level the quasi quark distribution captures all the collinear physics in the infinite momentum frame.

With the above results, we can construct a matching condition connecting the two distributions as

$$
\tilde{q}\left(x, \Lambda, P^{z}\right)=\int_{-1}^{1} \frac{d y}{|y|} Z\left(\frac{x}{y}, \frac{\Lambda}{P^{z}}, \frac{\mu}{P^{z}}\right) q(y, \mu)+\mathcal{O}\left(M^{2} /\left(P^{z}\right)^{2}\right)
$$

in the large $P^{z}$ limit, where the integration range is determined by the support of the quark distribution $q(y)$ on the light cone, the momentum fraction $x$ is defined in the finite momentum frame. We define the light-cone distribution $q(y, \mu)$ in the $\overline{\mathrm{MS}}$ scheme.

The matching factor $Z$ has a perturbative expansion in $\alpha_{s}$

$$
Z\left(\xi, \frac{\Lambda}{P^{z}}, \frac{\mu}{P^{z}}\right)=\delta(\xi-1)+\frac{\alpha_{s}}{2 \pi} Z^{(1)}\left(\xi, \frac{\Lambda}{P^{z}}, \frac{\mu}{P^{z}}\right)+\cdots
$$

and for $\xi>1$, one obtains

$$
Z^{(1)}(\xi) / C_{F}=\left(\frac{1+\xi^{2}}{1-\xi}\right) \ln \frac{\xi}{\xi-1}+1+\frac{1}{(1-\xi)^{2}} \frac{\Lambda}{P^{z}}
$$

whereas for $0<\xi<1$

$$
Z^{(1)}(\xi) / C_{F}=\left(\frac{1+\xi^{2}}{1-\xi}\right) \ln \frac{\left(P^{z}\right)^{2}}{\mu^{2}}+\left(\frac{1+\xi^{2}}{1-\xi}\right) \ln [4 \xi(1-\xi)]-\frac{2 \xi}{1-\xi}+1+\frac{\Lambda}{(1-\xi)^{2} P^{z}},
$$

and for $\xi<0$

$$
Z^{(1)}(\xi) / C_{F}=\left(\frac{1+\xi^{2}}{1-\xi}\right) \ln \frac{\xi-1}{\xi}-1+\frac{\Lambda}{(1-\xi)^{2} P^{z}}
$$

Near $\xi=1$, one has an additional term coming from the self energy correction

$$
Z^{(1)}(\xi)=\delta Z^{(1)}\left(2 \pi / \alpha_{s}\right) \delta(\xi-1)
$$

with

$$
\begin{aligned}
& \delta Z^{(1)}=\frac{\alpha_{S} C_{F}}{2 \pi} \int d y \\
& \quad \times \begin{cases}-\frac{1+y^{2}}{1-y} \ln \frac{y}{y-1}-1-\frac{\Lambda}{(1-y)^{2} P^{z}}, & y>1, \\
-\frac{1+y^{2}}{1-y} \ln \frac{\left(P^{z}\right)^{2}}{\mu^{2}}-\frac{1+y^{2}}{1-y} \ln [4 y(1-y)]+\frac{2 y(2 y-1)}{1-y}+1-\frac{\Lambda}{(1-y)^{2} P^{z}}, & 0<y<1, \\
-\frac{1+y^{2}}{1-y} \ln \frac{y-1}{y}+1-\frac{\Lambda}{(1-y)^{2} P^{z}}, & y<0,\end{cases}
\end{aligned}
$$

which provides a prescription for the singularity at $\xi=1$. The large logarithmic dependence on $P^{z}$ in $\tilde{q}\left(x, \Lambda, P^{z}\right)$ has been transformed into the renormalization scale dependence through the above matching condition. 
Up to now, we have considered only the quark contribution. One can start with an antiquark to do the one-loop calculation. In this case, one also has a contribution to $\tilde{q}\left(x, \Lambda, P^{z}\right)$ from $\bar{q}(x)$. However, the antiquark distribution has the property

$$
\bar{q}(x)=-q(-x),
$$

which is related to quark distribution at negative $x$. Also the $Z$ factor has the same property. After including both quark and antiquark contribution, the factorization Eq. (10) still applies, but now the quantities on the r.h.s. include also the antiquark contribution reflected by the negative $y$ region.

\section{Conclusion}

We discussed recent developments on computing parton distributions from their quasi counterparts, and presented the one-loop matching condition for the unpolarized non-singlet quark distribution. This matching condition connects the quasi quark distribution calculable on the lattice to the light-cone quark distribution measurable in experiments, thereby allows an extraction of the latter from the former.

\section{Acknowledgments}

This work was partially supported by the U. S. Department of Energy via grants DE-FG02-93ER-40762, and a grant (No. 11DZ2260700) from the Office of Science and Technology in Shanghai Municipal Government, and by National Science Foundation of China (No. 11175114, No. 11405104).

\section{References}

1. S. Alekhin, J. Blumlein and S. Moch, Phys. Rev. D 86, 054009 (2012) [arXiv:1202.2281 [hep-ph]].

2. J. Gao, M. Guzzi, J. Huston, H. L. Lai, Z. Li, P. Nadolsky, J. Pumplin and D. Stump et al., Phys. Rev. D 89, no. 3, 033009 (2014) [arXiv:1302.6246 [hep-ph]].

3. V. Radescu [H1 and ZEUS Collaboration], PoS ICHEP 2010, 168 (2010).

4. A. M. Cooper-Sarkar [ZEUS and H1 Collaborations], PoS EPS-HEP2011, 320 (2011) [arXiv:1112.2107 [hep-ph]].

5. A. D. Martin, W. J. Stirling, R. S. Thorne and G. Watt, Eur. Phys. J. C 63, 189 (2009) [arXiv:0901.0002 [hep-ph]].

6. R. D. Ball, V. Bertone, S. Carrazza, C. S. Deans, L. Del Debbio, S. Forte, A. Guffanti and N. P. Hartland et al., Nucl. Phys. B 867, 244 (2013) [arXiv:1207.1303 [hep-ph]].

7. X. Ji, J. H. Zhang and Y. Zhao, Phys. Rev. Lett. 111, 112002 (2013) [arXiv:1304.6708 [hep-ph]].

8. X. Ji, Phys. Rev. Lett. 110, 262002 (2013) [arXiv:1305.1539 [hep-ph]].

9. Y. Hatta, X. Ji and Y. Zhao, Phys. Rev. D 89, no. 8, 085030 (2014) [arXiv:1310.4263 [hep-ph]].

10. X. Xiong, X. Ji, J. H. Zhang and Y. Zhao, Phys. Rev. D 90, no. 1, 014051 (2014) [arXiv:1310.7471 [hep-ph]].

11. H. W. Lin, J. W. Chen, S. D. Cohen and X. Ji, arXiv:1402.1462 [hep-ph]. 
12. Y. Q. Ma and J. W. Qiu, arXiv:1404.6860 [hep-ph].

13. X. Ji, Sci. China Phys. Mech. Astron. 57, no. 7, 1407 (2014) [arXiv:1404.6680 [hep$\mathrm{ph}]]$.

14. X. Ji, P. Sun, X. Xiong and F. Yuan, arXiv:1405.7640 [hep-ph].

15. X. Ji, J. H. Zhang and Y. Zhao, arXiv:1409.6329 [hep-ph]. 